\title{
Transcriptome-Based Analysis Reveals Therapeutic Effects of Resveratrol on Endometriosis in aRat Model
}

\author{
Chunyan Wang ${ }^{1,2, *}$ \\ Zhengyun Chen ${ }^{1, *}$ \\ Xianlei Zhao ${ }^{2, *}$ \\ Cuicui Lin $^{2}$ \\ Shenghui Hong ${ }^{3}$ \\ Yuhan Lou ${ }^{2}$ \\ Xiaomeng Shi' \\ Mengdan Zhao' \\ Xiaohang Yang ${ }^{1,2}$ \\ Min-Xin Guan ${ }^{2}$ \\ Yongmei $\mathrm{Xi}^{1,2}$
}

'The Women's Hospital, Zhejiang University School of Medicine, Hangzhou, Zhejiang, 310006, People's Republic of China; ${ }^{2}$ Institute of Genetics, Zhejiang University; Department of Human Genetics, Zhejiang University School of Medicine, Zhejiang Provincial Key Laboratory of Genetic \& Developmental Disorders, Hangzhou, Zhejiang, 310058, People's Republic of China; ${ }^{3}$ Laboratory Animal Center of Zhejiang University, Hangzhou, Zhejiang, 31000I, People's Republic of China

*These authors contributed equally to this work

Correspondence: Yongmei Xi; Min-Xin Guan

Tel +86-057I-88206623; +86-057I$8898137 \mid$

Email xyyongm@zju.edu.cn; gminxin88@zju.edu.cn
Introduction: Endometriosis (EMs) is associated with severe chronic pelvic pain and infertility and the development of improved EMs treatment options is an ongoing focus. In this study, we investigated the effects of resveratrol on EMs and analyzed transcriptional changes in the lesions of model rats before and after resveratrol treatment.

Methods: We established arat model of endometriosis through the trans-implantation of endometrial fragments to the peritoneal wall and then used resveratrol as treatment. We then analyzed the results using RNA sequencing of the lesion tissues of each of the model rats before resveratrol treatment and the reduced lesion tissues after the treatment. Examinations of anatomy, biochemistry, immunohistochemical staining and flow cytometry examinations were also conducted. Other trans-implanted rats were also given sham treatments as shamtreatment control and other untrans-implanted rats served as sham-operation controls.

Results: In addition to the obvious lesions observed in the model rats, there were significant differences in the glucose tolerance, macrophage M1/M2 polarization, and adipocyte sizes between the treated model rats and sham (control) rats. Resveratrol treatment in the model rats showed significant efficacy and positive therapeutic effect. Transcriptional analysis showed that the effects of resveratrol on the endometriosis model rats were manifested by alterations in the PPAR, insulin resistance, MAPK and PI3K/Akt signaling pathways. Correspondingly, changes in PPAR $\gamma$ activation, M1/M2 polarization and lipid metabolism were also detected after resveratrol treatment.

Discussion: Our study revealed that resveratrol treatment displayed efficient therapeutic effects for EMs model rats, probably through its important roles in anti-inflammation, immunoregulation and lipid-related metabolism regulation.

Keywords: endometriosis, resveratrol, RNA-seq, glucose tolerance, PPAR $\gamma$

\section{Introduction}

Endometriosis (EMs) is acommon benign gynecological condition characterized by endometrial-like tissue occurring outside the uterus. ${ }^{1}$ It is estimated that $10-15$ women of reproductive age suffer from endometriosis. ${ }^{2}$ Provisional treatment mainly consists of surgery and/or hormonal medication, which are often associated with side effects and of variable efficacy. ${ }^{3}$ It has been proposed that several of these factors, including coelomic metaplasia, immune system abnormalities, genetic causes, environmental factors and lifestyle factors, might account for the development of this disease. ${ }^{4}$ Nevertheless, the etiology and pathogenesis of endometriosis remain uncertain, thus hindering the development of treatment options. Long-term 
treatments of greater efficacy than those currently available are highly coveted for this condition.

Natural compounds such as resveratrol, epigallocatechin-3-gallate, curcumin, puerarin, ginsenosides, xanthohumol, 4-hydroxybenzyl alcohol, quercetin, apigenin have been considered and initially attempted for the treatment of endometriosis using animal models or cell lines. ${ }^{5,6}$ Among them, resveratrol, aphytoalexin polyphenol found in natural plants or fruits, is comparably easier to obtain. As anatural ingredient found in unprocessed foods, resveratrol is also considered safe, in contrast to some of the above competing compounds that are more readily classified as herbal medicines. Resveratrol also benefits from abackground of comparatively extensive studies with many of its associated pathways elucidated, such as PPAR and MARK signaling. ${ }^{7}$ Such aspects are far less developed for most of the other compounds or substances listed. Resveratrol, has previously been highlighted as apotential supplement for the treatment of cancers, ${ }^{8}$ cardiovascular disease ${ }^{9}$ and EMs. ${ }^{10}$ Pharmacological effects of resveratrol on the energy and lipid metabolism have been highlighted in animal models, particularly related to the human eutopic endometrial stromal cells (HESCs) of EMs. ${ }^{11}$ The highlighted benefits relating to lipid metabolism and other aspects in this study contain little overlap with findings from the other potential compounds.

Resveratrol shows great potential for the treatment of endometriosis, particularly relating to avariety of physiological and therapeutic effects. ${ }^{12}$ Adecrease in the number and volume of endometrial implants; in suppressed proliferation, vascularization, inflammation, cell survival, and in an increase in apoptosis. ${ }^{13-16}$ In vitro studies showed that resveratrol reduced the invasiveness of endometrial stromal cells and suppressed their inflammatory responses. ${ }^{17-}$ 19 In clinical trials, resveratrol enhanced the role of oral contraceptives in the treatment of endometrium-associated dysmenorrhea by decreasing endometrium-aromatase and cyclooxygenase- 2 expression. ${ }^{10}$ In another randomized exploratory trial, the effects of resveratrol were assessed positively for angiogenesis as well as upon inflammation in the eutopic endometrium. ${ }^{20}$ Overall, the use of resveratrol as anoninvasive and effective therapy for endometriosis is expected. However, the mechanisms of Ems remain incompletely understood.

With the development of high-throughput sequencing, RNA-seq provides an approach for diagnosis and for the elucidation of the pathology of endometriosis. It has already been used to analyze the transcriptome differences between eutopic and ectopic endometrial specimens from endometriosis patients, ${ }^{21,22}$ the oocytes of endometriosis patients and healthy controls, ${ }^{23}$ and for the macrophage polarization in women's endometrium with or without disease. ${ }^{24}$ RNA-seq was also used to compare the menstrual effluent of patients with and without endometriosis for investigating its pathobiology and biomarkers of endometriosis. $^{25}$

Activated macrophages are usually divided into two categories: M1 and M2 macrophages. ${ }^{26}$ Both M1 and M2 macrophages are closely related to inflammatory responses. Among these M1 macrophages are involved in pro-inflammatory responses. By contrast, M2 macrophages are mainly involved in anti-inflammatory responses and in the repair of damaged tissues. ${ }^{27,28}$ Endometriosis has been considered as "a disease of the macrophage", 26 which is characterized by ahigher level of macrophage infiltration. ${ }^{29-31}$ In the immune dysregulation that is characteristic of endometriosis, M1 and M2 macrophage polarization of the peritoneal fluid are noted as elevated in the endometriosis groups, ${ }^{32,33}$ with ectopic endometrial tissue exhibiting inflammatory characteristics which may be involved in the overall immune response.

It has been reported that low body mass index (BMI) and unfavorable serum lipid profiles are often in evidence in patients with EMs. ${ }^{34}$ Patients with aBMI $<18.5 \mathrm{~kg} / \mathrm{m}^{2}$ are more likely to have asevere EMs phenotype. ${ }^{35}$ Higher volumes of adipocytes are negatively associated with lipolysis. ${ }^{36}$ The enlargement of adipocyte cells may be related to lipid metabolism disorder and inflammatory response. ${ }^{36}$ In addition, PPAR $\gamma$ plays an important role in directly or indirectly affecting the development of glucose tolerance and of the polarization of macrophages in different disease phenotypes that relate to both lipid metabolism and chronic inflammation. ${ }^{37,38}$ This seems to be an indicator that there may be some strong links between disorders of fat digestion and the absorption and activation of macrophages in cases of EMs.

In this study, we used resveratrol to treat model rats and analyzed transcriptome differences of the lesions before and after treatment. We also assessed the polarization of macrophages, insulin resistance, glucose tolerance, and adipocytes in the EMs rat models before and after treatment. Changes in PPAR $\gamma$ activation, M1/M2 polarization and lipid metabolism were detected after the resveratrol treatment. Our data suggests that the use of resveratrol may provide significant anti-inflammation, immunoregulation and lipidrelated metabolism mitigation for endometriosis. 


\section{Materials and Methods}

\section{Establishment of aRat Model of EMs}

Fifty female Sprague Dawley rats aged 8-10 weeks, weighing 200-250 g, were placed in aclean-level environment in the Zhejiang University Laboratory Animal Center. Animal experimental methods and purposes were all in line with the Laboratory Animal-Guideline for Ethical Review of Animal Welfare (GB/T 35892-2018) as issued by the Zhejiang University Experimental Animal Welfare Ethics Review Committee. Rats having a4-5day estrous cycle and two consecutive estrus cycles were then selected for surgery. Considering the potential postoperative pain level of this small incision was classified as "mild to moderate", welfare nursing was provided after the operation. If post-operative signs of more than expected and transient pain or distress were observed, appropriate intervention strategies were adopted including analgesics, anesthetics, and/or euthanasia. During the operation, aseptic conditions for the operation were adhered to, and 3\% Pentobarbital (BIOCAM) was selected for anesthesia. Then rat estrus epithelial tissue with a $0.8 \times 0.8 \mathrm{~cm}^{2}$ endometrium was auto-transplanted into the endometrial abdominal wall. Ten rats were also selected for aplacebo operation to serve as sham. All animals were fed regularly for 4 weeks before the laparotomy examination.

\section{Lesion Examination and Measuring}

The rats were anesthetized and laparotomy was performed to measure the size of the implant. Modeled rats were recorded and the lesion volume was calculated using thefollowing formula: $V=a \times b^{2} / 2$ (where arepresents the broadest transverse diameter of the lesion, brepresents the vertical diameter line) and $\mathrm{V} \geq 2 \mathrm{~mm}^{3}$ was considered as asuccessful model. Considering the number, strength, and distribution of lesion adhesions, we also measured the focal adhesions score: $0=$ no adhesion; $1=$ thin filmy adhesions; $2=$ more than one thin adhesion; $3=$ thick adhesion with focal point; $4=$ thick adhesion with planar attachment; $5=$ very thick vascularized adhesion or more than one planar adhesion. ${ }^{39}$

\section{Resveratrol Treatment}

Resveratrol was dissolved in 35\% DMSO for intraperitoneal injection in rats, while the Sham group and EMs group were injected with the same amount of the solvent $(0.9 \% \mathrm{NaCl}+35 \mathrm{DMSO})$. Thirty rats exhibiting successful modelling were divided into three groups according to lesion size: EMs group $(\mathrm{n}=10$, with equal resveratrol solvent), Res-med group ( $\mathrm{n}=10$, resveratrol dose $15 \mathrm{mg} /$ $\mathrm{Kg} / \mathrm{d}$ ), Res-high group ( $\mathrm{n}=10$, resveratrol dose $=45 \mathrm{mg}$ / $\mathrm{Kg} / \mathrm{d}$ ), the control group was the Sham-operation group (Sham, $n=10$, with equal solvent amount). The rats of four groups were administered continuously for 28 days. Lesions were examined (as the above method). Lesion tissues were sampled before and after resveratrol treatment for evaluation.

\section{RNA Extraction and Library Preparation}

Total RNA was extracted from the implanted endometrium from the EM group and Res-high group. mRNA was purified from the total RNA using poly-T oligo-attached magnetic beads. Fragmentation was carried out using divalent cations under elevated temperature in the Fragmentation Buffer, where first strand cDNA was synthesized using fragmented mRNA, random hexamer primers and M-MuLC reverse transcriptase. Second strand cDNA was subsequently synthesized using DNA polymerase Iand dNTP. Remaining overhangs were converted into blunt ends. After adenylation of $3^{\prime}$ ends of DNA fragments, adaptors with hairpin loop structure were ligated to prepare for hybridization. In order to select cDNA fragments of preferentially $370-420 \mathrm{bp}$ in length, the library fragments were purified with AMPure XP systemm(Beckman Coulter, Beverly, USA), and then PCR was performed. PCR products were purified and library quality was assessed on the Agilent Bioanalyzer 2100 system (Agilent Technologies, CA, USA).

\section{RNA-Seq Analysis}

Raw reads of fastq format were firstly processed through in-house perl scripts. Clean data was obtained by removing reads containing adaptors, reads containing Nbases and low-quality reads from raw data. Paired-end clean reads were aligned to the reference genome using Hisat2 v2.0.5 Before analysis of differentially expressed genes we firstly performed unsupervised hierarchical clustering and obtained aheat map. We applied the DESeq2 algorithm to filter the differentially expressed genes between the groups. The resulting P-values were adjusted using Benjamini and Hochberg's approach for controlling false discovery rates. Genes with an adjusted Padj (adjusted pvalue) $<0.05$, fold change of (FC) 1.5 found by DESeq2 were assigned as differentially expressed. Avolcano plot was then constructed showing the differentially expressed genes. 


\section{Enrichment Analysis of Differentially Expressed Genes}

Gene Ontology (GO) enrichment analysis of differentially expressed genes was implemented by the clusterProfiler Rpackage, in which gene length bias was corrected. GO terms with corrected P-values less than 0.05 were considered significantly enriched as differentially expressed genes. Pathway analysis was applied to determine the significant pathways of differentially expressed genes according to the Kyoto Encyclopedia of Genes and Genomes (KEGG). The DO (Disease Ontology) database describes the function of human genes and diseases. The DisGeNET database integrates human disease-related genes. We used clusterProfiler Rpackage or software to test the statistical enrichment of differential expression genes in KEGG pathways, the DO pathway and the DisGeNET pathway. Pathways with corrected P-values less than 0.05 were considered significantly enriched by differentially expressed genes.

\section{Glucose Tolerance Test (GTT)}

Before the GTT test, rats were all subject to an overnight fasting of 16 hours, from $5 \mathrm{pm}$. to $9 \mathrm{am}$. After overnight fasting, $20 \%$ glucose solution was administered to rats orally at adosage of $0.01 \mathrm{~mL} / \mathrm{g}$ body weight. Blood samples were collected from the tail vein before and 30, 60, 90 and $120 \mathrm{~min}$ after glucose administration and blood glucose level was determined using ablood glucose meter (ROCHE).

\section{Insulin Tolerance Test (ITT)}

Before the ITT test, rats were all subject to afasting of 4 hours. After fasting, diluted human insulin (Novolin R) was administered to rats by intraperitoneal injection at adosage of 0.5 unit $/ \mathrm{kg}$ body weight. Blood samples were collected from the tail vein before and 15, 30, 60, 90 and $120 \mathrm{~min}$ after insulin injection and blood glucose levels were determined using ablood glucose meter (ROCHE).

\section{Flow Cytometry}

After euthanizing the rats, peritoneal cells were retrieved by peritoneal lavage with PBS buffer. After shaking the rat, peritoneal cells were collected. In this study, CD 68 and CD 206 were used as M1 and M2 macrophage markers, respectively. ${ }^{40}$ The peritoneal macrophages were fixed with $2 \%$ paraformaldehyde for 10 min before incubation with anti-rat CD 68 antibody (1:100, A13286, ABclonal) and anti-rat CD 206 antibody (1:100, A8301,
ABclonal). After washing with PBS buffer, the cells were resuspended in PBS buffer for flow cytometry analysis. ${ }^{41}$

\section{HE Staining and Immunohistochemistry}

Tissues were fixed in 10\% formalin and dehydrated with agradient of alcohol for paraffin slicing. The paraffinembedded sections were processed according to astandard protocol for staining with Hematoxylin and Eosin (Solarbio). Briefly, sections were dewaxed and rehydrated and stained with hematoxylin. After differentiation, sections were stained with eosin. For immunohistochemical staining on ectopic endometrium lesions, slices were heated in an EDTA buffer (pH9.0) to retrieve antigens. Then to block endogenous peroxide, slices were put in 3\% $\mathrm{H}_{2} \mathrm{O}_{2}$ for $20 \mathrm{~min}$. After blockage with normal serum, the diluted primary antibody PPAR $\gamma \quad(1: 100$, A0270, ABclonal) was applied and incubated overnight at $4{ }^{\circ} \mathrm{C}$. The nextday, slices were treated with HRP-conjugated secondary antibody, visualized with diaminobenzidine (DAB) and counterstained with hematoxylin. Images were taken under alight microscope (Nikon) and pathological features were analyzed. Adipose cell size was calculated using ImageJ. Brown dots were viewed as positive signals.

\section{Quantitative Real-Time Polymerase Chain Reaction (RT-PCR)}

Total RNA was extracted from endometrial implants using Trizol reagent (Invitrogen, 15596026) and reverse transcribed using PrimeScriptTM RT Reagent Kit (Takara, RR037A). Quantitative real-time PCR was performed on 7900 HT Fast Real-time PCR System (Applied Biosystems) using TB Green Premix Ex TaqTM (Takara, RR420A). Experiments were repeated in triplicate and data was analyzed using the 7900 System SDS RQ Manager software, and relative gene expression was determined using the $2-\Delta \Delta \mathrm{Ct}$ method by normalization to the GAPDH levels. Primer sequences for this study are listed in Supplemental Table1.

\section{Statistical Analysis}

Statistical analysis was performed using GraphPad PRISM (GraphPad Software Inc., CA, USA). All data was presented as mean \pm SEM. Comparisons between groups were performed using one-way analysis of variance (ANOVA) followed by Dunnett's multiple comparison 
test. In all comparisons, $\mathrm{P}<0.05$ was considered to be statistically significant.

\section{Results}

\section{Endometriotic Lesions Size Assessment and Treatment of Resveratrol in Rat Models}

Intraperitoneal endometriotic lesions were surgically induced by suturing uterine tissue samples to the abdominal wall. We carried out histological analyses on estrous cycles in rats to determine the transplantation period (Figure1A). Figure1B shows that uterine tissue samples, which were sutured to the abdominal wall of rats models without any myometrial tissue (Figure1B). A0.8 $\times 0.8 \mathrm{~cm}^{2}$ segment was ligated with apolypropylene 4-0 suture (Figure1C).

After modeling for 4 weeks, the cystic structured endometriotic implants were examined by anesthesia and laparotomy. The transplanted uterine tissue samples had developed to typical endometriotic lesions consisting of cyst-like dilated endometrial glands and vascular stroma. We observed highly different lesion volumes varying from $2 \mathrm{~mm}^{3}$ to over $100 \mathrm{~mm}^{3}$. In modelling, $27 \%$ of the rats failed to develop as adequate models, probably due to the timing of modeling being out of the estrous cycle. Successful model rats were then treated with resveratrol for 4 weeks. All model rats appeared healthy with no obvious side effects. We then dissected and measured the cyst volume of the reduced lesions, and compared them with the size and adhesion of the lesions before treatment. There were significant differences before and after the resveratrol treatment (Figure1D-G).

\section{Differential Gene Expression Analysis Before and After Resveratrol Treatment}

With RNA microarray hybridization, 22,159 clones hybridized with probes were detected with positive signals (Supplemental Table2). DESeq2 analysis showed that, compared with the EMs group, 2123 genes were at least 1.5 fold differentially expressed (adjusted p-value $<0.05$ ) in the ectopic lesions of the Res treatment group, in which 910 were downregulated and 1213 were upregulated (Figure2A and B). Among down-regulated genes, some of them come to our particular attention, including F13a1, acoagulation factor; ${ }^{42} \mathrm{Hbb}$, aregulator of pain and inflammation; ${ }^{43}$ Tiam1, aregulator of cell invasion, ${ }^{44} \mathrm{Msr1}$, involved in macrophage-associated physiological and pathological processes, ${ }^{45} \mathrm{Cd} 5$ and Mcp1, associated with the immunoregulatory and inflammatory processes. ${ }^{46}$ Among up-regulated genes, Mir145 has been reported to be related to MicroRNAs in cancer and DNA damage response; ${ }^{47}$ and Crhr2 has been shown to promote the activation of adenylate cyclase, leading to increased intracellular cAMP levels. ${ }^{48}$

Differentially expressed genes (DEGs) between EMs group and Res group were screened using the lima package followed by exclusion of DEGs with the genefilter package. Enrichment analysis was performed on DEGs using AmiGO2, the top 50 DEGs are shown in the heatmap (Figure2C).

\section{KEGG and Gene Ontology Analysis}

A total of 61 pathways were significantly enriched with differentially expressed genes, in which 22 pathways were downregulated and 39 were upregulated after resveratrol treatment $($ Padj $<0.05$; Figure3A and B). These changes were mainly related to metabolic and immunity pathways, such as PPAR and MAPK signaling. In addition, these upand downregulated genes were enriched with GO term enrichment. Results showed that upregulated genes were enriched in blood vessel morphogenesis, angiogenesis, transmembrane transport and similar (Figure3C). Conversely, the downregulated genes were related to regulation of leukocyte mediated immunity and of leukocyte activation (Figure3D).

\section{Effects of Resveratrol Manifested by Increased Glucose Tolerance and Enlarged Adipocytes}

In our previous study, we analyzed the effects of resveratrol treatment on human ectopic endometrial stromal cells. ${ }^{49}$ Here, lipidomics analysis showed that resveratrol altered lipid profiles and resulted in changes in glycerophospholipid metabolism pathways and in the activation of insulin resistance. To further confirm, we performed examinations of GTT and ITT, adipocyte histology and flow cytometry of the peritoneal fluid in the EMs rats (Figure4A). Surprisingly, EMs rats showed atrend toward glucose intolerance showing an increase in glucose levels at $60 \mathrm{~min}$ and $90 \mathrm{~min}$ after injection (Figure4B), as well as insulin resistance in EMs rats only 15- min after injection (Figure4C), compared with controls. Regarding treatment, the Res-high group exhibited ameliorated glucose intolerance (vs.controls), compared with sham-treated EMs rats injected with glucose (vs.control) (Figure4D). There were no significant differences in ITT among each group after resveratrol treatment. (Figure4E). 
A

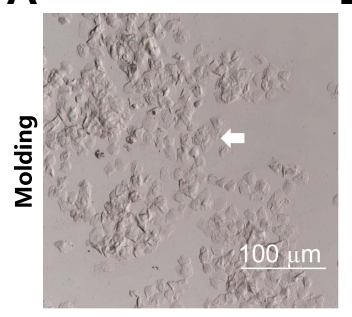

D EMs
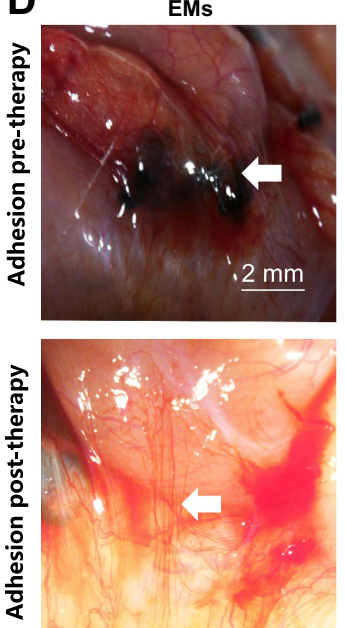

$\mathbf{F}$
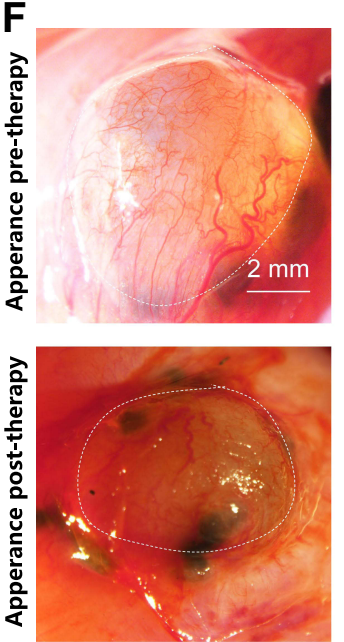

B
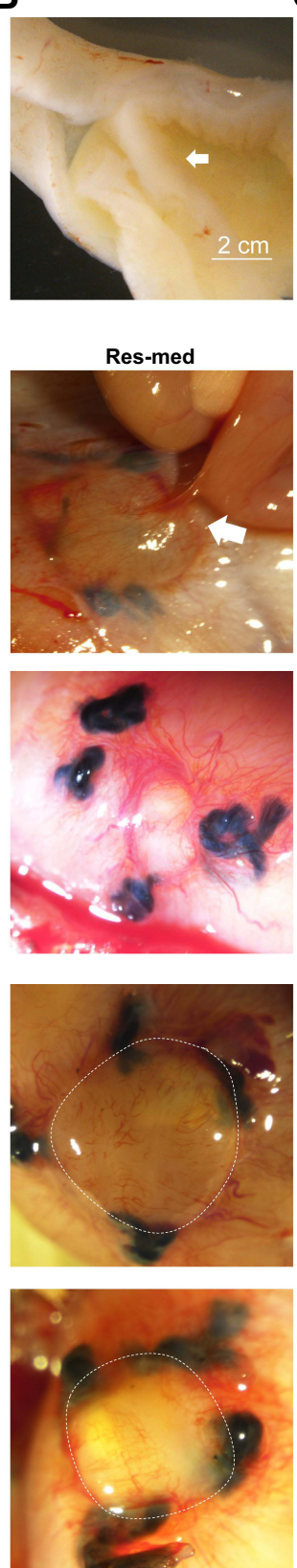

C
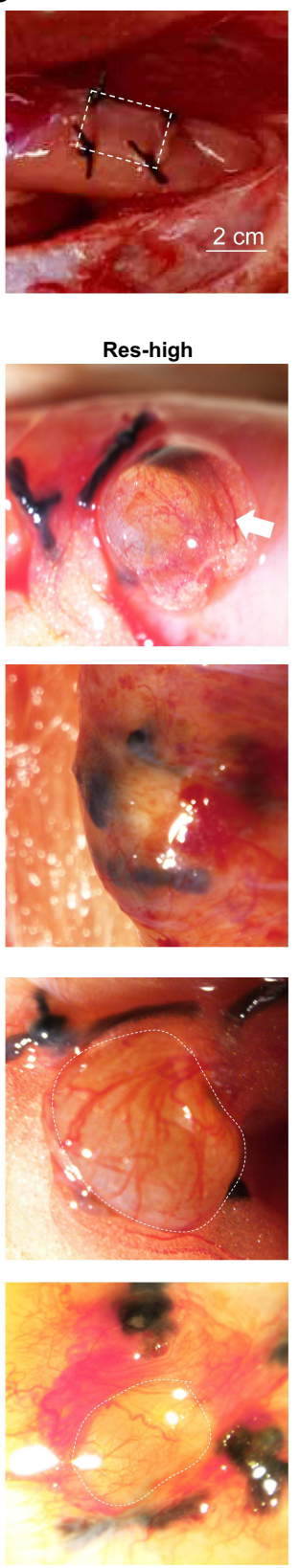

E

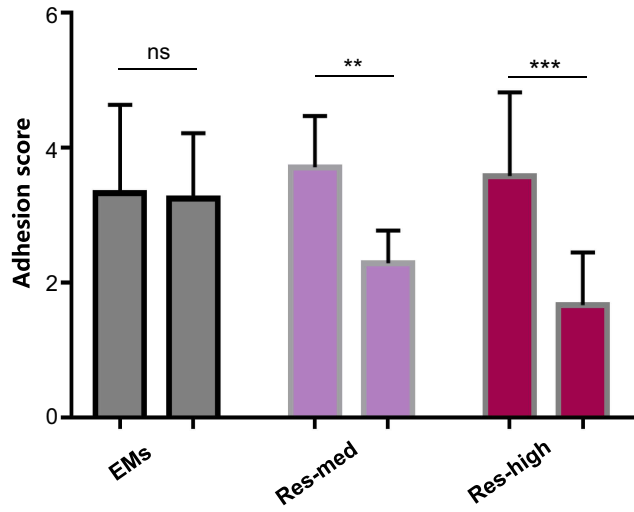

G

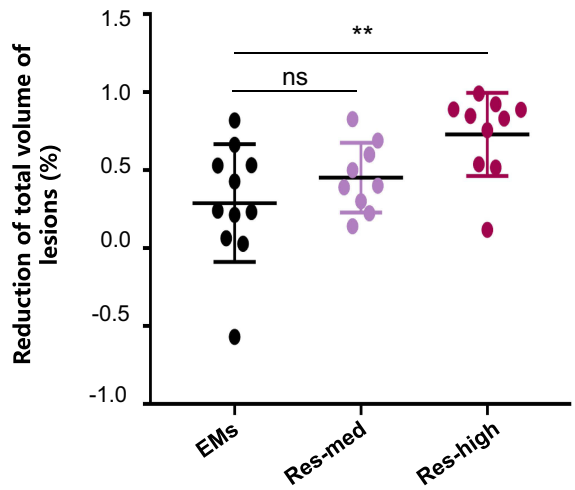

Figure I Molding display and effects of resveratrol on the lesions of model rats. (A) The estrous cycle stages of female rats were examined using vaginal biopsy samples. Arrow: Irregular keratinocyte like cells gathered together. Scale bars: $100 \mu \mathrm{m}$. (B) Rat estrus epithelial tissue. Arrows indicate correctly attached to the abdominal suture. Arrow: Rat estrus epithelial tissue which separation from myometrium of uterus. Scale bars: $2 \mathrm{~cm}$. (C) Rat estrus epithelial tissue was auto-transplanted into the endometrial abdominal wall. Dotted lines: Estrus epithelial tissue of rat endometrium transplanted to the abdominal wall. Scale bars: $2 \mathrm{~cm}$. Morphology and volume reduction of the implants were determined after 28 days of treatment. Scale bars: $2 \mathrm{~cm}$. (Dand E) Extent of reduction of endometrial-like lesion adhesions. Arrows indicate adhesion. Scale bar: $2 \mathrm{~mm}$. (Fand G) Reduction of endometrial-like lesion volume. Dotted lines indicate the volume of lesions. Scale bar: $2 \mathrm{~mm}$. Data are presented as mean \pm SEM, $* * \mathrm{P}<$ 0.01 , ***P $<0.001$ EMs vs control (ANOVA).

Abbreviations: Res-med, dose $=15 \mathrm{mg} / \mathrm{Kg} / \mathrm{d}$; Res-high, dose $=45 \mathrm{mg} / \mathrm{Kg} / \mathrm{d}$; ns, no significance.

In human patients, women with endometriosis have alower BMI and areduced number of adipocyte cells than those without this disease. ${ }^{50,51}$ It is interesting, we found an increased adipocyte size in EMs rats and asignificantly reduced adipocyte size after resveratrol treatment (Figure4F-G).
Resveratrol Induced Changes in $\mathrm{MI}$ and M2 Macrophage Polarization and in

\section{Inflammatory Cytokines}

By using flow cytometry and two established markers for M1 and M2 macrophage polarity, CD68 and CD 206 
A

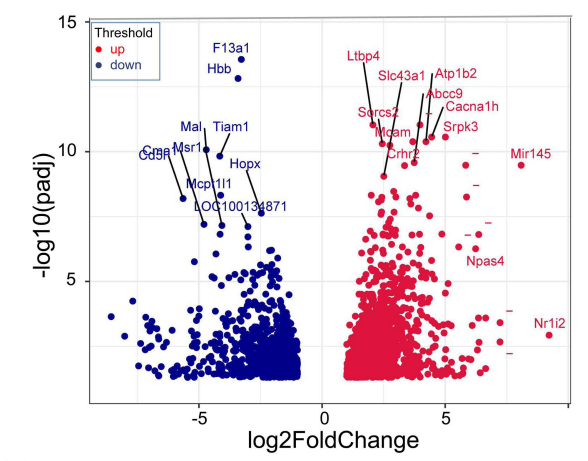

B

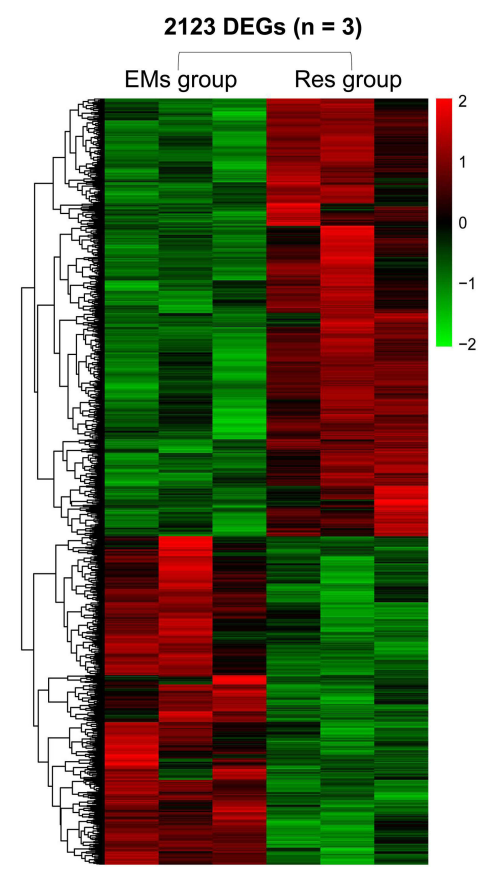

C

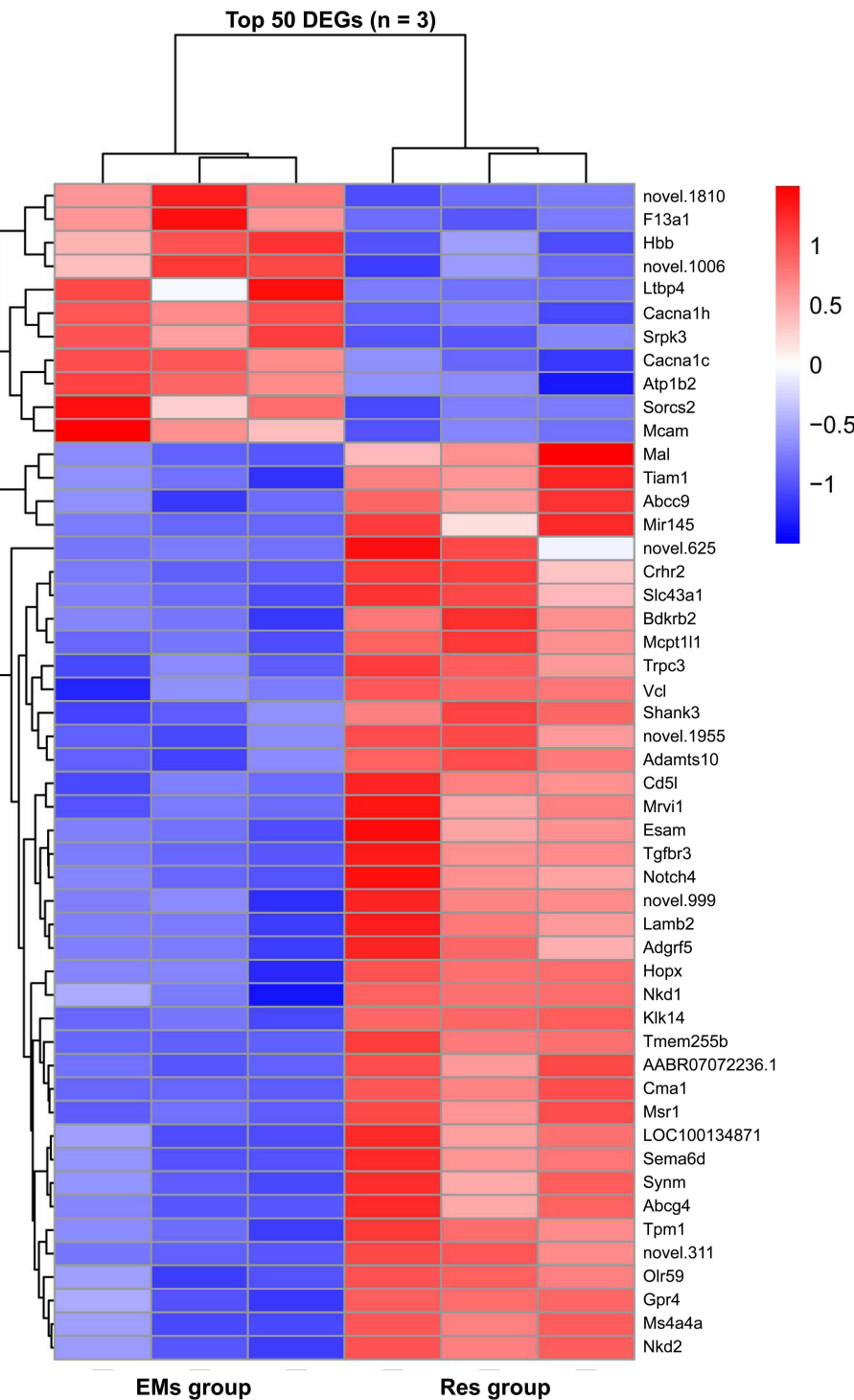

Figure2 Differential gene expression analysis before and after resveratrol treatment. (A) Volcano plots of differentially regulated genes between Res-high treatment and EMs group. Red indicates upregulated, while blue indicates downregulated expression ( $=3$ EMs group, $n=3$ Res-high group, FC $\geq 1.5$, Padj $<0.05$ ). (B) Heatmap of differentially expressed genes (2I23 probes) between EMs group and Res-high group. Each column represents asingle sample, and each row represents an RNA For each group $n=3$. (C) Heatmap of the top 50 differentially expressed genes (DEGs) between EMs group and Res-high group expression profiles. Three lines of each group were analyzed $(n=3)$.

respectively, we examined M1 and M2 macrophages in peritoneal fluid of the model rats before and after resveratrol treatment and analyzed the relative ratios of $\mathrm{M} 1$ and M2 (Figure5A). Results showed that M1/M2 was elevated in the EMs group and significantly reduced after the Reshigh dose treatment (Figure5B). To characterize the changes in inflammatory cytokines upon resveratrol treatment, we analyzed the mRNA levels of IL-10, which is an anti-inflammatory cytokine and of INF- $\gamma$, IL- 6 and TNF $\alpha$, which are pro-inflammatory cytokines. Corresponding to the M1/M2 reduction, IL10 was increased in the Res groups (Figure5C), whilst INF- $\gamma$, IL-6 and TNF $\alpha$ were correspondingly decreased (Figure5D-F). This might correspond to the changes in M1 and M2 macrophages polarization during anti-inflammation mechanisms upon resveratrol treatment. The RNA-seq analysis data provides us adeeper understanding of these phenotypic characteristics.

\section{Resveratrol Induced PPAR $\gamma$ Activation in Ectopic Focus of EMs Rats}

Insulin resistance initially occurs in the adipose tissue and PPAR $\gamma$ plays an important role in adipose tissue differentiation, macrophage polarization, and adipocytokine 
A

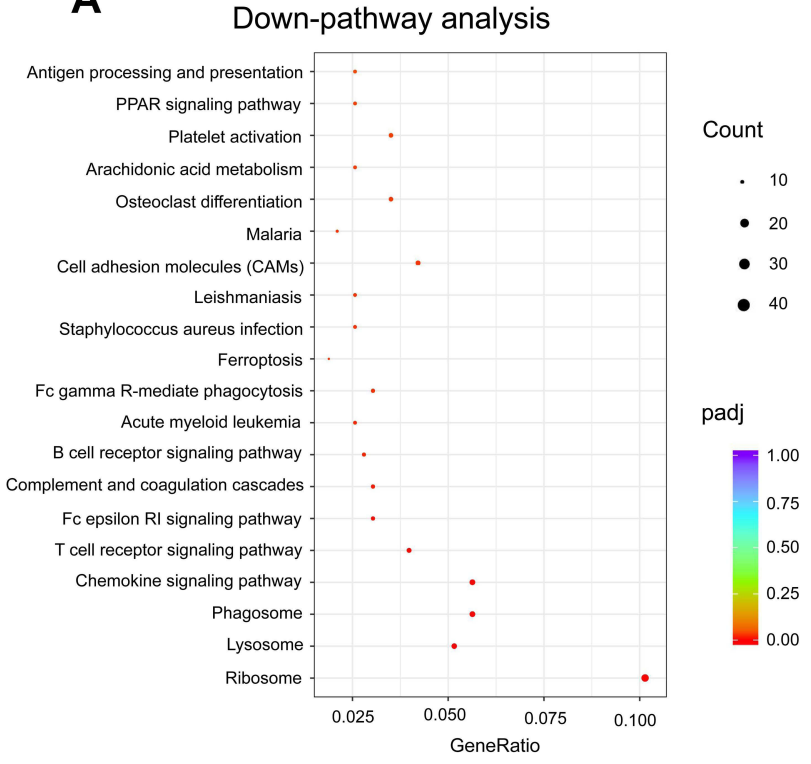

C

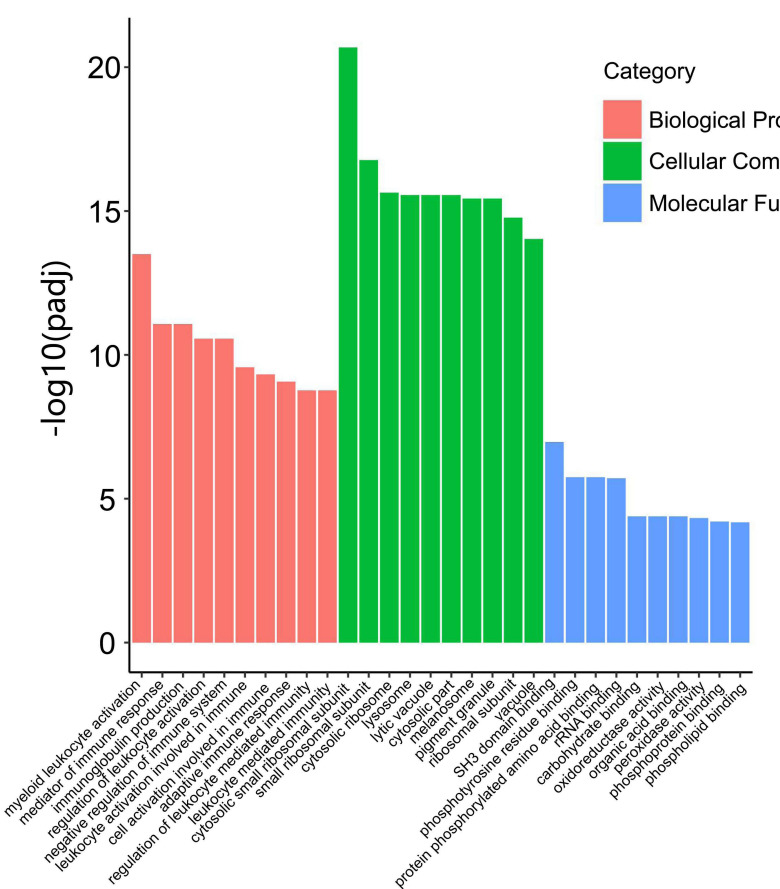

B

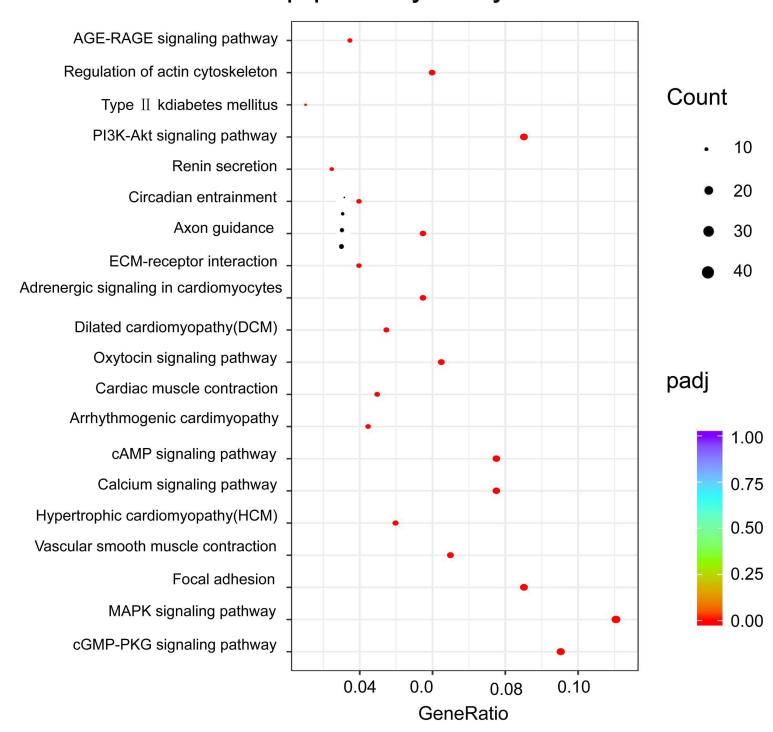

D

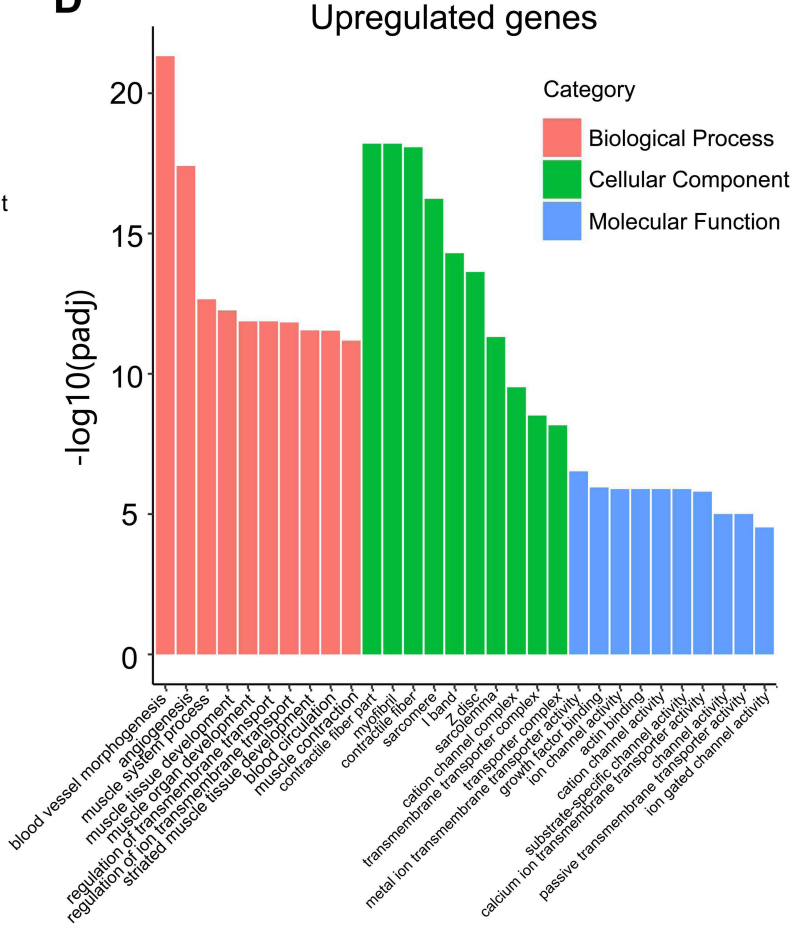

Figure3 Pathway enrichment analysis. (Aand B) KEGG pathway analysis: Functional analysis for the significantly down-regulated genes (A) and up-regulated (B) genes. The vertical axis showed the annotated functions of the target genes. The horizontal axes shows the enrichment score (gene ratio) and the gene number of each cluster. Only the top 20 significantly enriched clusters are included. (Cand D) GO terms based on cellular component, molecular function, and biological process of the upregulated and downregulated genes.

secretion. ${ }^{37,38,50}$ We analyzed the PPAR $\gamma$ expression in model rats. Scattered weak staining of PPAR $\gamma$ expression in stromal cells was found in EMs group (Figure 6A-D). Decreased PPAR $\gamma$ expression was shown in the untreated
EMs groups compared with the controls. High dose resveratrol treatment induced intense staining of PPAR $\gamma$ expression in glandular epithelial cells in the lesion tissues of model rats. The surface epithelium was markedly atrophic 


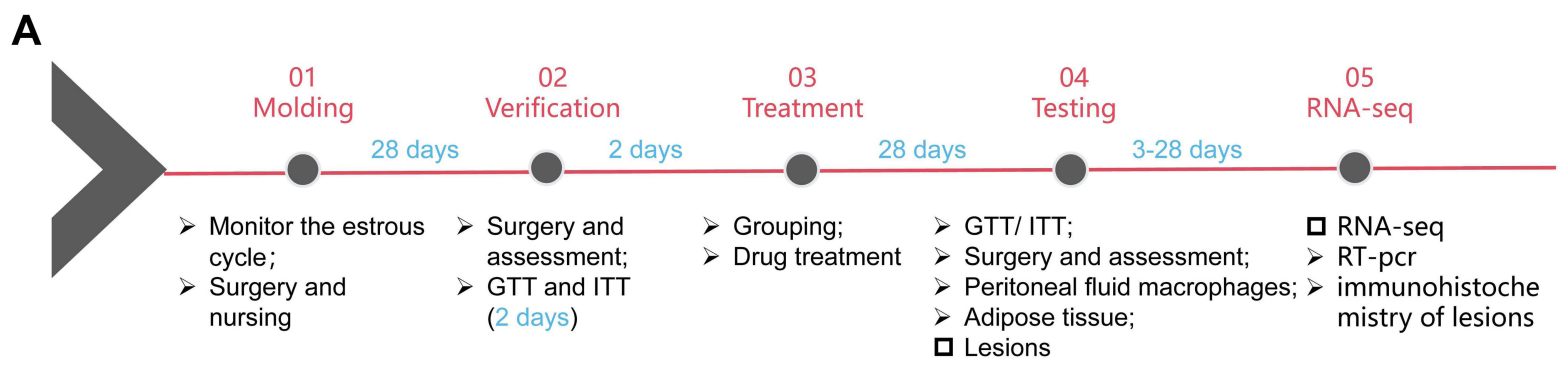

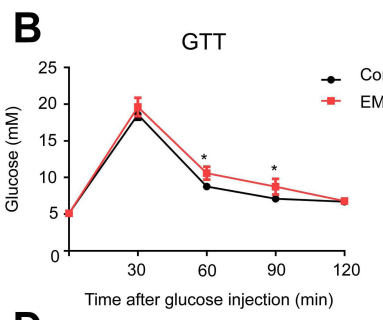

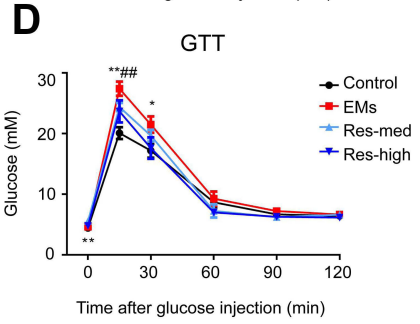

$\mathbf{F}$

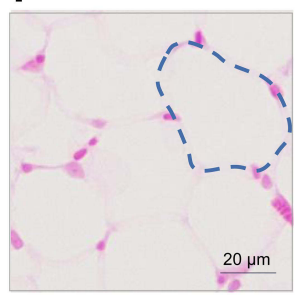

Control

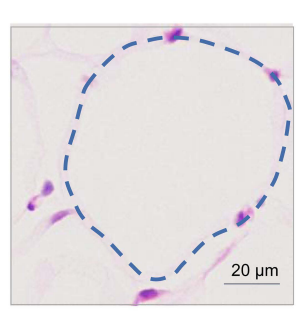

EMs
AUC

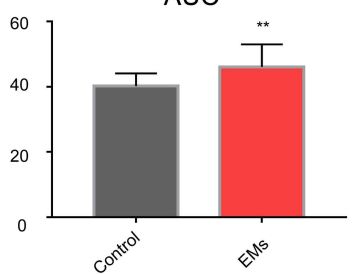

AUC

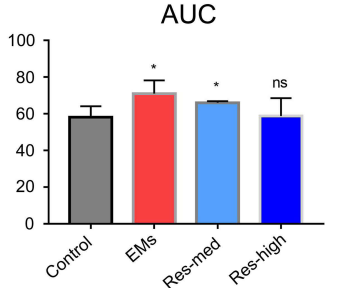

C

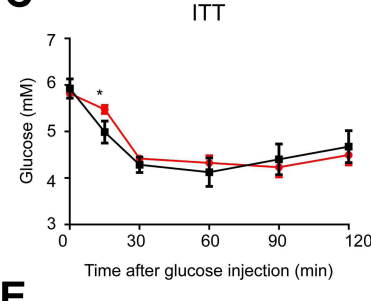

ITT
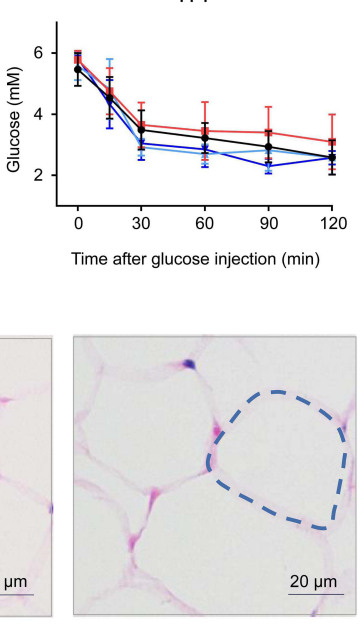

Res-high
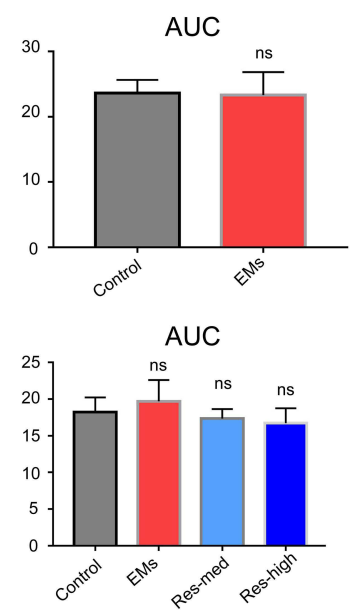

G

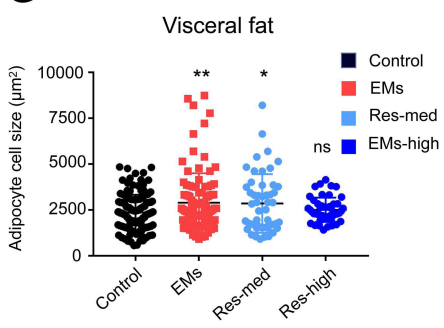

Figure4 Effects of resveratrol manifested by ameliorated glucose tolerance and enlarged adipocytes. (A) Experimental design flow chart. (B-E) Plasma glucose levels are shown inmg/dl for rat models ( $n=6-9$ for each group) after glucose administration for glucose tolerance test (GTT) ((Band D), panels on the left) or after insulin administration for insulin tolerance test (ITT) ((Cand E), panels on the left). Areas under GTT or ITT glucose curves were calculated per I20 minutes, for EMs and for controls, respectively ((B-E), panels on the right). Plasma glucose levels were measured for aliquots of GTT or ITT samples from 0, 30-, 60-, 90- or I20-min time points. (F) Histology of gonadal adipose tissue from rat models. Scale bar $20 \mu \mathrm{m}$. (G) Statistical image of adipocyte size in gonadal adipose tissue. Data are presented as mean \pm SEM, *P $<0.05$; ${ }^{* * P}<0.01$ EMs vs control, ${ }^{\#} \mathrm{P}<0.0$ l Res-high vs control (ANOVA).

Abbreviations: Res-med, dose $=15 \mathrm{mg} / \mathrm{Kg} / \mathrm{d}$; Res-high, dose $=45 \mathrm{mg} / \mathrm{Kg} / \mathrm{d}$; ns, no significance.

and no staining could be found in the Res-high group although weak staining was observed in the EMs group and Res-med group (Figure6B and C). PPAR $\gamma$ expression was improved after resveratrol treatment and higher expressions were shown in the Res-high group compared to the Res-med group (Figure6D and E). The mRNA expression of PPAR $\gamma$ was significantly increased in both Res-med and Res-high (vs Control group), with significant differences between Res-med and EMs groups $(\mathrm{P}<0.01)$ (Figure 6F).

\section{Discussion}

Endometriosis is characterized by the ectopic presence of endometrial-like tissue (glands and stroma) outside the uterine cavity, especially in the pelvic area. ${ }^{51}$ Over recent decades, the prevalence of endometriosis has been reported to ranges from as low as $1.9 \%$ to as high as $20.7 \%$ of the susceptible female population. ${ }^{52-54}$ Meanwhile, it has anegative impact on quality of life and imposes aheavy financial burden on patients. The therapeutic effect of resveratrol on EMs rats, involving proliferation, apoptosis, oxidative stress, inflammation, invasion 


\section{A}
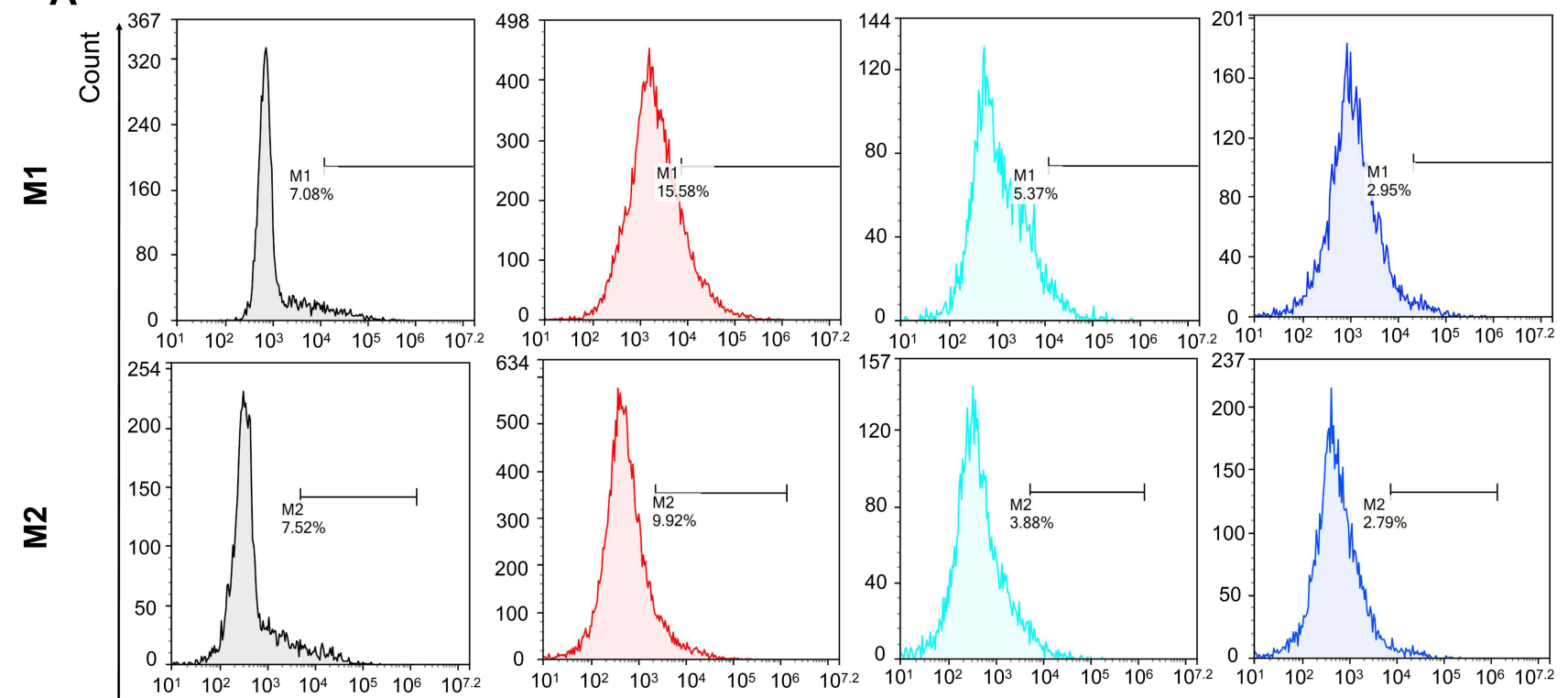

Control

EMs

Res-med

Res-high

FITC

B

Macrophage in peritoneal fluid

C

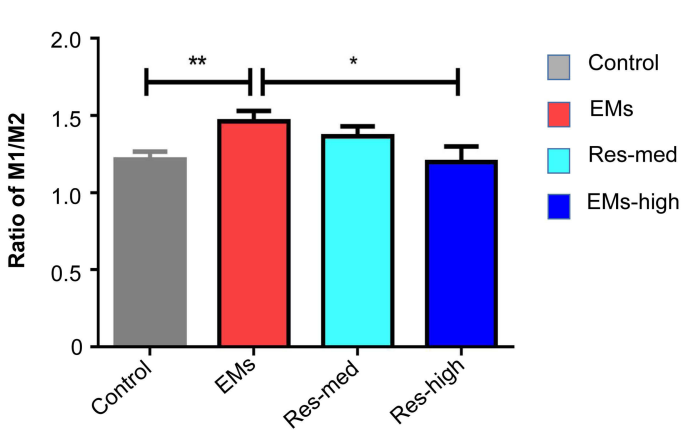

E

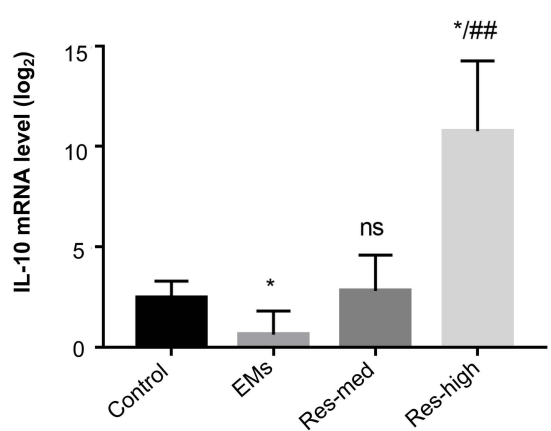

F
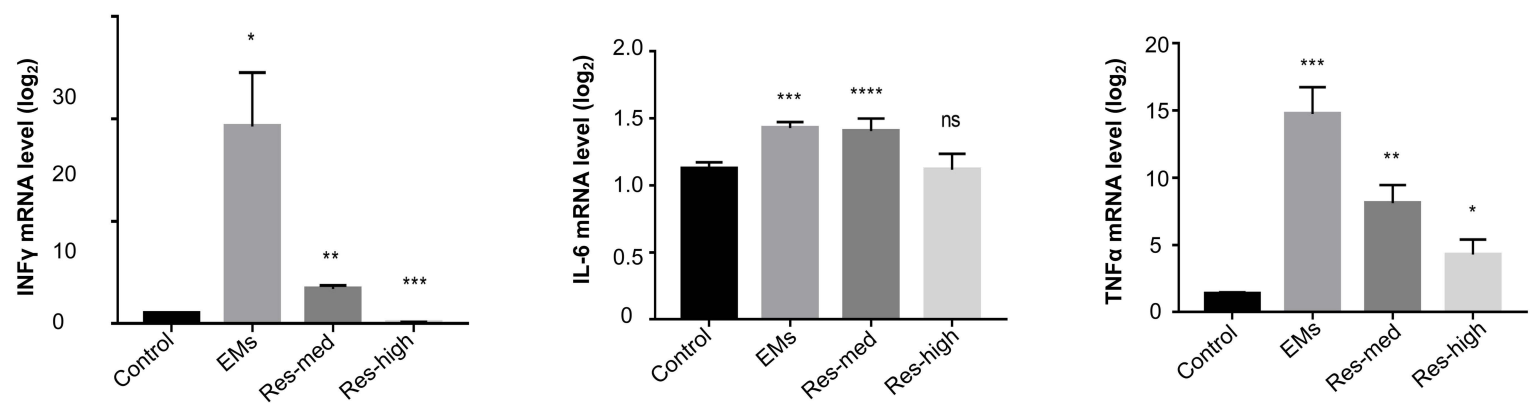

Figure5 Examination of changes in MI and M2 macrophages polarization and inflammatory cytokines. (A) Flow Cytometric analysis of macrophage in peritoneal fluid from endometriosis, resveratrol treatment and control subjects CD 68 and CD $206(n=6-10)$. (B) Statistical analysis of macrophage MI/M2 in peritoneal fluid. (C-F). mRNA levels of IL-10 (C), IFN $\gamma(\mathbf{D})$, IL-6 (E) and TNF $\alpha(\mathbf{F})$ were analyzed in ectopic endometrial lesions and upon resveratrol treatment. GAPDH was used as aloading control. Data are presented as mean $\pm \mathrm{SEM},{ }^{*} \mathrm{P}<0.05 ; * * \mathrm{P}<0.01$, ***P $<0.00$ I, ****P $<0.000$ I EMs vs control; ${ }^{\# *} \mathrm{P}<0.01$ Res-high vs control (ANOVA).

Abbreviations: Res-med, dose $=15 \mathrm{mg} / \mathrm{Kg} / \mathrm{d} ;$ Res-high, dose $=45 \mathrm{mg} / \mathrm{Kg} / \mathrm{d}$; ns, no significance.

and angiogenesis have been suggested in anumber of publications. ${ }^{55-57}$ In this study, we focused on the transcriptomic changes in EMs rats upon resveratrol treatment and emphasize that resveratrol regulates lipid the metabolism, adipocyte function and also relieves the inflammatory response of endometriosis. 
A

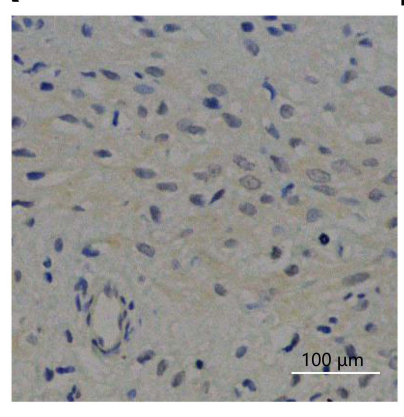

Control
B

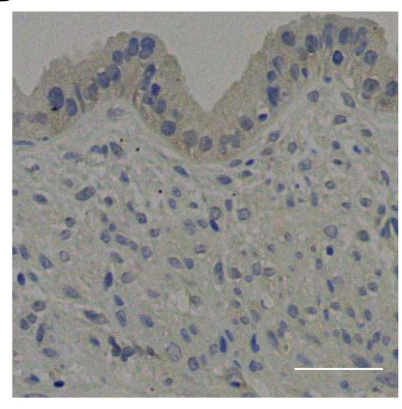

EMs
C

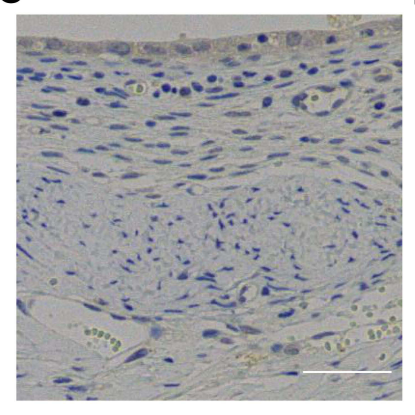

Res-med
D

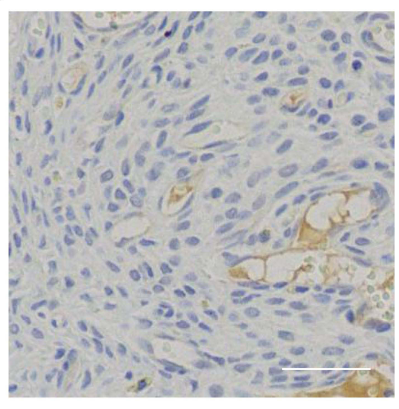

Res-high
E

Expression of PPARy in ectopic lesions

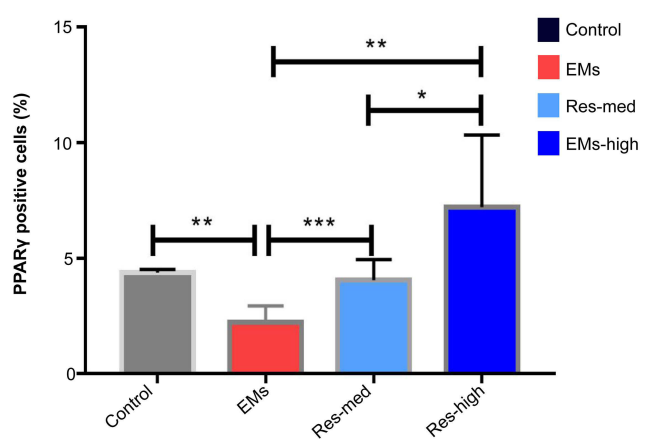

F

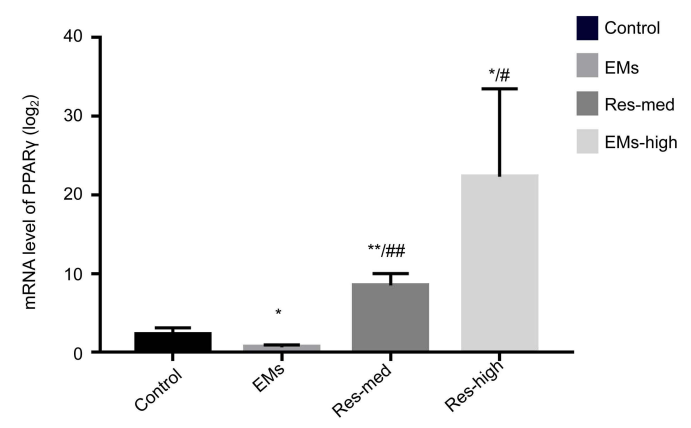

Figure6 Resveratrol induced PPAR $\gamma$ activation in ectopic focus of EMs rats. (A-D) Immunohistological staining of PPAR $\gamma$ expression. PPAR $\gamma$ expression in stromal cells in Control (A), EMs group (B), Res-med group (C) and Res-high group (D) as well as weak staining in surface epithelial cells in EMs group (B), Res-med group (C). Strong staining of PPAR $\gamma$ expression in glandular epithelial cells was showed in the lesion tissues of model rats after resveratrol treatment compared to the untreated EMs groups (D). (E) Immunohistological PPAR $\gamma$ expression levels were semiquantitatively analyzed by counting of positive and total cell number of the epithelial and stromal cells. The percentages of positive cells/total cells are shown. (F) Effects of resveratrol on PPAR $\gamma$ mRNA expression. PPAR $\gamma$ mRA transcripts normalized to $\beta$-actin expression were quantified in real-time. Scale bar: $100 \mu \mathrm{m}$. Data are presented as mean $\pm \mathrm{SEM}$. ${ }^{*} \mathrm{P}<0.05$; ${ }^{* * \mathrm{P}}<0.0 \mathrm{I}$ and ${ }^{* * * P}<0.00 \mathrm{I} E M s$ vs control; ${ }^{\#} \mathrm{P}<0.05$, ${ }^{\# \#} \mathrm{P}<0.0 \mathrm{I}$ Res-high vs control (ANOVA).

Although high-throughput sequencing data analysis provide us with alot of information, we particularly considered the low-scoring pathways focused on metabolism, and immunity as potential validation. In our experiment, after resveratrol treatment, in addition to the decreased lesion size of rat models, we detected some broader improved phenotypes such as those relating to macrophage polarization, adipocyte size, or glucose tolerance. We then attempted to draw more relevant information from RNAseq data analysis towards any related mechanisms of EMs.

Insulin resistance not only occurs in obesity, but also in lipodystrophy. ${ }^{58}$ The white adipocyte is exquisitely sensitive to insulin, lipolytic activity and insulin sensitivity occur with notable variance between different compartments of adipose tissues. ${ }^{58}$ Here we choose the gonadal adipose tissues near the ectopic foci. We found that adipocyte sizes were increased in EMs. It has been reported that ahigher volume of adipocytes is associated negatively with lipolysis. ${ }^{36}$ Our study revealed that resveratrol altered the macrophage polarization, glucose tolerance, and decreased adipocyte size in amanner that may inhibit the initiation and progression of endometriosis.

The polarization of macrophages, glucose tolerance, and higher volume of adipocyte are all influenced by PPAR $\gamma .{ }^{37,38}$ We have suggested that resveratrol may mitigate the above phenotypes via the activation of PPAR $\gamma$. When comparing before and after resveratrol treatment, differentially expressed genes were related to metabolic, immunoregulatory and inflammatory processes, including the PPAR signaling pathway and MAPK signaling pathway (Figure7). The PPARs are critical regulators of the lipid-associated metabolism and PPAR $\gamma$ has been proposed as apotential therapy for endometriosis, especially in angiogenesis. ${ }^{59}$ The MAPKs signaling pathway is asignal transduction system that mediates extracellular signals to intracellular reactions. ${ }^{60}$ MAPK could be the hub of the response pathway upon resveratrol stimulation. Therefore, MAPKs could function as pivotal intracellular signal 
transducers in endometriotic cells which relate to inflammatory changes.

We noticed that among the down regulated factors of the PPAR signaling pathway, Cyp27a1, Scd, Fabp4, Plin2, Slc27a6, Cpt1b, Rxra, Fabp5, Cpt1c, Acs15, Angpt14, Dbi, Pdpk1, Fabp1 were all decreased, whilst PPAR $\gamma$ was not. In addition to PPAR $\gamma$ histochemical staining of the lesion tissue, we also examined mRNA levels of PPAR $\gamma$ in the lesion samples before and after resveratrol treatment. This did show an increase in PPAR $\gamma$ after resveratrol treatment. Our findings support arole for resveratrol in the pathophysiology of endometriosis, and provide new targets for investigation into possible causes and treatments. In the present study, limitations remain, including the relatively small sample size for transcriptome sequencing, difficulties in elucidating insulin resistance associated with endometriosis rats and identifying the M1 and M2 macrophages. Further investigations are also required for the validation of molecular pathways involved in resveratrol treatment.

\section{Conclusions}

Our findings extend the analytic results of RNA levels in the EMs rat models treated with resveratrol, thereby nominating specific transcriptional and signaling pathways as therapeutic targets for this gynecological condition that is distressing and difficult to treat. We assessed the polarization of macrophages, glucose tolerance, and adipocytes in the EMs rat models before and after the treatment. Changes in PPAR $\gamma$ activation, M1/M2 polarization and the lipid metabolism were all detected after the resveratrol treatment. We conclude that resveratrol may play roles in

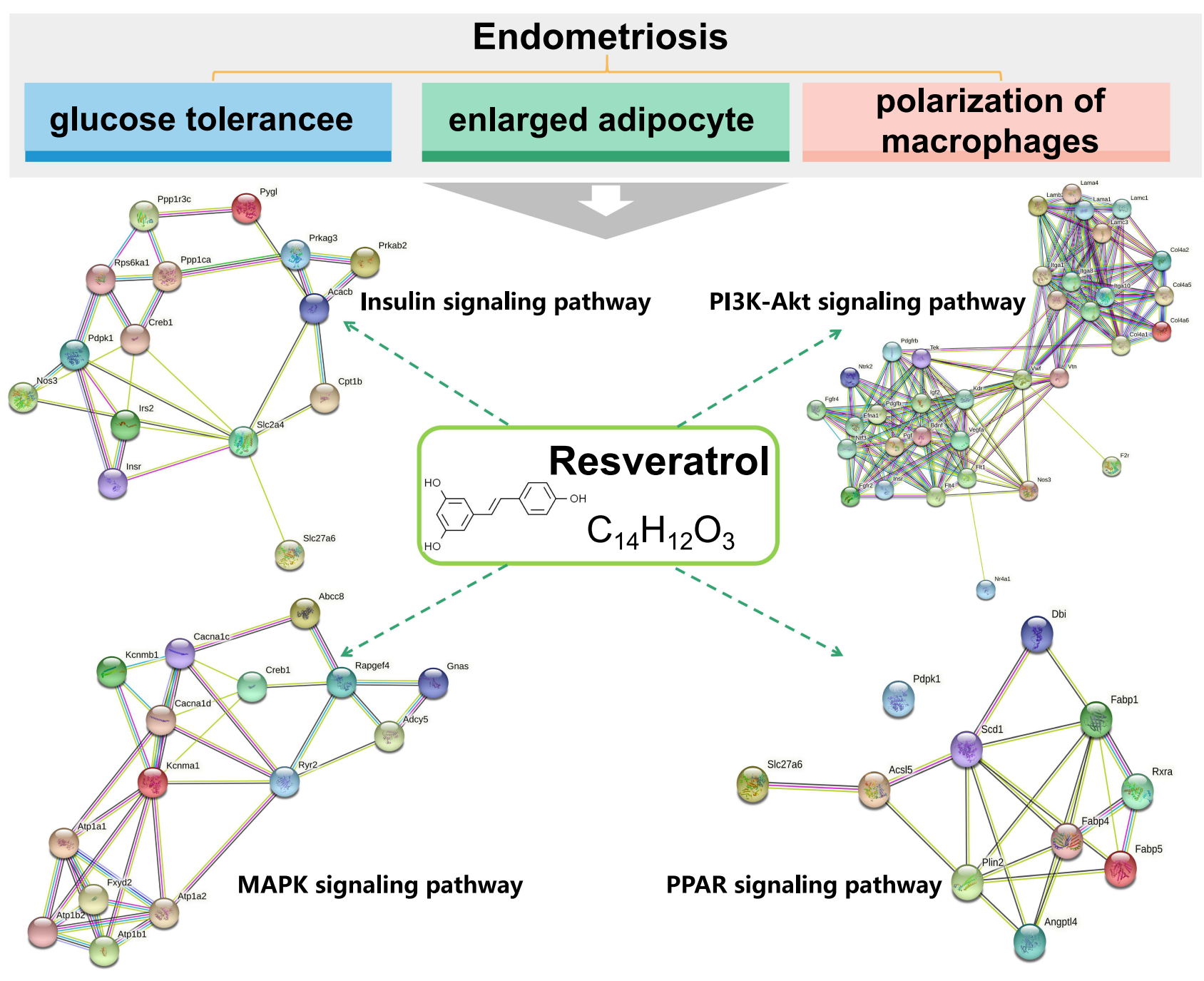

Figure7 Schematic representation of the significant pathways of resveratrol in animal models of Endometriosis. Gene interaction network among the differential genes from STRING protein-protein interaction networks using the protein coding genes of transcriptomics data. Nodes represent direct acting proteins. Edges with different color styles represent different acting types. 
anti-inflammation, immunoregulation and the lipid-related metabolism in the treatment of endometriosis.

\section{Abbreviations}

EMs, endometriosis; Padj, adjusted pvalue; FC, fold change; KGEE, Kyoto Encyclopedia of Genes and Genomes; GTT, glucose tolerance test; ITT, insulin tolerance test; MAPK, mitogen-activated protein kinases.1; SEM, standard error of mean; PPARs, peroxisome proliferators-activated receptors; AKT, protein kinase B.

\section{Ethics in Publishing}

All animal experiments were approved by the Zhejiang University Experimental Animal Welfare Ethics Review Committee. The treatment and welfare of animals strictly followed the Laboratory Animal-Guideline for Ethical Review of Animal Welfare (GB/T 35892-2018) issued by the Zhejiang University Experimental Animal Welfare Ethics Review Committee.

\section{Acknowledgments}

We are very grateful to Chris Wood from the Life Science College of Zhejiang University for language support. Thanks to Liuping and Feiyu Feng for providing help in the raising experimental animals in the Laboratory Animal Center of Zhejiang University.

\section{Author Contributions}

All authors made asignificant contribution to the work reported, whether that is in the conception, study design, execution, acquisition of data, analysis and interpretation, or in all these areas; took part in drafting, revising or critically reviewing the article; gave final approval of the version to be published; have agreed on the journal to which the article has been submitted; and agree to be accountable for all aspects of the work.

\section{Funding}

This work was funded by The National Key R\&D Program of China (2018YFC1004900, 2018YFC1003 201), Natural Science Foundation of China (grant 82071616), Zhejiang National Science Foundation (LGF20H040010, LY17H040004, LY19H040011).

\section{Disclosure}

The authors have declared that no conflict of interest exists.

\section{References}

1. VercelliniP, ViganòP, SomiglianaE, etal. Endometriosis: pathogenesis and treatment. Nat Rev Endocrinol. 2014;10(5):261-275. doi:10.1038/nrendo.2013.255

2. ShafrirAL, FarlandLV, ShahDK, etal. Risk for and consequences of endometriosis: acritical epidemiologic review. Best Pract Res Clin Obstet Gynaecol. 2018;51:1-15. doi:10.1016/j.bpobgyn.2018.06.001

3. GiudiceLC. Clinical practice. Endometriosis. NEngl JMed. 2010;362 (25):2389-2398. doi:10.1056/NEJMcp1000274

4. MehedintuC, PlotogeaMN, IonescuS, AntonoviciM. Endometriosis still a challenge. JMed Life. 2014;7(3):349-357.

5. HalpernG, SchorE, KopelmanA. Nutritional aspects related to endometriosis. Rev Assoc Med Bras (1992). 2015;61(6):519-523. doi:10.1590/1806-9282.61.06.519

6. MeresmanGF, GötteM, LaschkeMW. Plants as source of new therapies for endometriosis: areview of preclinical and clinical studies. Hum Reprod Update. 2021;27(2):367-392. doi:10.1093/humupd/dmaa039

7. ParkHS, LimJH, KimMY, etal. Resveratrol increases AdipoR1 and AdipoR2 expression in type 2 diabetic nephropathy. JTransl Med. 2016;14(1):176. doi:10.1186/s12967-016-0922-9

8. HuminieckiL, HorbańczukJ. The functional genomic studies of resveratrol in respect to its anti-cancer effects. Biotechnol Adv. 2018;36(6):1699-1708. doi:10.1016/j.biotechadv.2018.02.011

9. PrysyazhnaO, WolhuterK, SwitzerC, etal. Blood pressure-lowering by the antioxidant resveratrol is counterintuitively mediated by oxidation of cGMP-dependent protein kinase. Circulation. 2019;140 (2):126-137. doi:10.1161/CIRCULATIONAHA.118.037398

10. Mendes da SilvaD, GrossLA, NetoEDPG, etal. The use of resveratrol as an adjuvant treatment of pain in endometriosis: arandomized clinical trial. JEndocr Soc. 2017;1(4):359-369. doi:10.1210/js.201700053

11. Kolahdouz MohammadiR, ArablouT. Resveratrol and endometriosis: invitro and animal studies and underlying mechanisms (Review). Biomed Pharmacother. 2017;91:220-228. doi:10.1016/j. biopha.2017.04.078

12. Kolahdouz-MohammadiR, ShidfarF, KhodaverdiS, etal. Resveratrol treatment reduces expression of MCP-1, IL-6, IL-8 and RANTES in endometriotic stromal cells. JCell Mol Med. 2021;25(2):1116-1127. doi: $10.1111 / \mathrm{jcmm} .16178$

13. Rudzitis-AuthJ, MengerMD, LaschkeMW. Resveratrol is apotent inhibitor of vascularization and cell proliferation in experimental endometriosis. Hum Reprod. 2013;28(5):1339-1347. doi:10.1093/ humrep/det031

14. ErgenoğluAM, YenielAÖ, ErbaşO, etal. Regression of endometrial implants by resveratrol in an experimentally induced endometriosis model in rats. Reprod Sci. 2013;20(10):1230-1236. doi:10.1177/ 1933719113483014

15. Bayoglu TekinY, GuvenS, KirbasA, etal. Is resveratrol apotential substitute for leuprolide acetate in experimental endometriosis? Eur JObstet Gynecol Reprod Biol. 2015;184:1-6. doi:10.1016/j.ejogrb. 2014.10.041

16. YavuzS, AydinN, CelikO, etal. Resveratrol successfully treats experimental endometriosis through modulation of oxidative stress and lipid peroxidation. JCancer Res Ther. 2014;10(2):324-329. doi:10.4103/0973-1482.136619

17. VillanuevaJA, SokalskaA, CressAB, etal. Resveratrol potentiates effect of simvastatin on inhibition of mevalonate pathway in human endometrial stromal cells. JClin Endocrinol Metab. 2013;98(3): E455-E462. doi:10.1210/jc.2012-3387

18. TaguchiA, KogaK, KawanaK, etal. Resveratrol enhances apoptosis in endometriotic stromal cells. Am JReprod Immunol. 2016;75 (4):486-492. doi:10.1111/aji.12489

19. TaguchiA, Wada-HiraikeO, KawanaK, etal. Resveratrol suppresses inflammatory responses in endometrial stromal cells derived from 
endometriosis: apossible role of the sirtuin 1 pathway. JObstet Gynaecol Res. 2014;40(3):770-778. doi:10.1111/jog.12252

20. KhodarahmianM, AmidiF, MoiniA, etal. Arandomized exploratory trial to assess the effects of resveratrol on VEGF and TNF- $\alpha 2$ expression in endometriosis women. JReprod Immunol. 2021;14 3:103248. doi:10.1016/j.jri.2020.103248

21. ChenS, LuoY, CuiL, etal. miR-96-5p regulated TGF- $\beta /$ SMAD signaling pathway and suppressed endometrial cell viability and migration via targeting TGFBR1. Cell Cycle. 2020;19(14):1740-1753. doi:10.1080/15384101.2020.1777804

22. WangD, LuoY, WangG, etal. Circular RNA expression profiles and bioinformatics analysis in ovarian endometriosis. Mol Genet Genomic Med. 2019;7(7):e00756. doi:10.1002/mgg3.756

23. FerreroH, CorachánA, AguilarA, etal. Single-cell RNA sequencing of oocytes from ovarian endometriosis patients reveals adifferential transcriptomic profile associated with lower quality. Hum Reprod. 2019;34(7):1302-1312. doi:10.1093/humrep/dez053

24. Vallvé-JuanicoJ, SantamariaX, VoKC, etal. Macrophages display proinflammatory phenotypes in the eutopic endometrium of women with endometriosis with relevance to an infectious etiology of the disease. Fertil Steril. 2019;112(6):1118-1128. doi:10.1016/j.fertnstert. 2019.08.060

25. WarrenLA, ShihA, RenteiraSM, etal. Analysis of menstrual effluent: diagnostic potential for endometriosis. Mol Med. 2018;24(1):1. doi:10.1186/s10020-018-0009-6

26. MartinezFO, GordonS. The M1 and M2 paradigm of macrophage activation: time for reassessment. F1000Prime Rep. 2014;6:13. doi:10.12703/P6-13

27. IvashkivLB. Epigenetic regulation of macrophage polarization and function. Trends Immunol. 2013;34(5):216-223. doi:10.1016/j.it.20 12.11.001

28. MurrayPJ, AllenJ, BiswasS, etal. Macrophage activation and polarization: nomenclature and experimental guidelines. Immunity. 2014;41(1):14-20. doi:10.1016/j.immuni.2014.06.008

29. SmithSR, LovejoyJC, GreenwayF, etal. Contributions of total body fat, abdominal subcutaneous adipose tissue compartments, and visceral adipose tissue to the metabolic complications of obesity. Metabolism. 2001;50(4):425-435. doi:10.1053/meta.2001.21693

30. TordjmanJ, DivouxA, PriftiE, etal. Structural and inflammatory heterogeneity in subcutaneous adipose tissue: relation with liver histopathology in morbid obesity. JHepatol. 2012;56(5):1152-1158. doi:10.1016/j.jhep.2011.12.015

31. GolanR, ShelefI, RudichA, etal. Abdominal superficial subcutaneous fat: aputative distinct protective fat subdepot in type 2 diabetes. Diabetes Care. 2012;35(3):640-647. doi:10.2337/dc11-1583

32. NieMF, XieQ, WuY-H, etal. Serum and ectopic endometrium from women with endometriosis modulate macrophage M1/M2 polarization via the Smad2/Smad3 pathway. JImmunol Res. 2018;20 18:6285813. doi: $10.1155 / 2018 / 6285813$

33. LaganàAS, SalmeriFM, Ban FrangežH, etal. Evaluation of M1 and M2 macrophages in ovarian endometriomas from women affected by endometriosis at different stages of the disease. Gynecol Endocrinol. 2020;36(5):441-444. doi:10.1080/09513590.2019.1683821

34. LiuY, ZhangW. Association between body mass index and endometriosis risk: a meta-analysis. Oncotarget. 2017;8(29):46928-46936. doi:10.18632/oncotarget.14916

35. Lafay PilletMC, SchneiderA, BorgheseB, etal. Deep infiltrating endometriosis is associated with markedly lower body mass index: a476 case-control study. Hum Reprod. 2012;27(1):265-272. doi:10.1093/ humrep/der346

36. CristanchoAG, LazarMA. Forming functional fat: agrowing understanding of adipocyte differentiation. Nat Rev Mol Cell Biol. 2011;12 (11):722-734. doi:10.1038/nrm3198

37. LiZY, SongJ, ZhengS-L, etal. Adipocyte metrnl antagonizes insulin resistance through PPAR $\gamma$ signaling. Diabetes. 2015;64(12):40 11-4022. doi:10.2337/db15-0274
38. OdegaardJI, Ricardo-GonzalezRR, GoforthMH, etal. Macrophagespecific PPARgamma controls alternative activation and improves insulin resistance. Nature. 2007;447(7148):1116-1120. doi:10.1038/ nature 05894

39. HeringtonJL, GloreDR, LucasJA, etal. Dietary fish oil supplementation inhibits formation of endometriosis-associated adhesions in achimeric mouse model. Fertil Steril. 2013;99(2):543-550. doi:10. 1016/j.fertnstert.2012.10.007

40. Garaicoa-PazminoC, FretwurstT, SquarizeCH, etal. Characterization of macrophage polarization in periodontal disease. JClin Periodontol. 2019;46(8):830-839. doi:10.1111/jcpe.13156

41. SunH, LiD, YuanM, etal. Macrophages alternatively activated by endometriosis-exosomes contribute to the development of lesions in mice. Mol Hum Reprod. 2019;25(1):5-16. doi:10.1093/molehr/gay049

42. GemmatiD, ViglianoM, BuriniF, etal. Coagulation factor XIIIA (F13A1): novel perspectives in treatment and pharmacogenetics. Curr Pharm Des. 2016;22(11):1449-1459. doi:10.2174/138161282 2666151210122954

43. AnMY, EoH, SonH, etal. Anti-inflammatory effects of leaf and branch extracts of honeyberry (Lonicera caerulea) on lipopolysaccharide-stimulated RAW264.7 cells through ATF3 and Nrf2/HO-1 activation. Mol Med Rep. 2020;22(6):5219-5230. doi:10.3892/mmr. 2020.11638

44. DiamantopoulouZ, WhiteG, FadlullahMZH, etal. TIAM1 antagonizes TAZ/YAP both in the destruction complex in the cytoplasm and in the nucleus to inhibit invasion of intestinal epithelial cells. Cancer Cell. 2017;31(5):621-634.e6. doi:10.1016/j.ccell.2017.03.007

45. TangY, LiH, LiJ, etal. Macrophage scavenger receptor 1 contributes to pathogenesis of fulminant hepatitis via neutrophil-mediated complement activation. JHepatol. 2018;68(4):733-743. doi:10.1016/j. jhep.2017.11.010

46. RhodesNP, HuntJA, WilliamsDF. Macrophage subpopulation differentiation by stimulation with biomaterials. JBiomed Mater Res. 1997;37(4):481-488. doi:10.1002/(SICI)1097-4636(19971215)37: 4<481::AID-JBM6>3.0.CO;2-H

47. SuzukiHI, YamagataK, SugimotoK, etal. Modulation of microRNA processing by p53. Nature. 2009;460(7254):529-533. doi:10.1038/ nature 08199

48. TsudaT, TakefujiM, WettschureckN, etal. Corticotropin releasing hormone receptor 2 exacerbates chronic cardiac dysfunction. JExp Med. 2017;214(7):1877-1888. doi:10.1084/jem.20161924

49. ZhengyunC, Chunyan W, Cuicui L, et al. The Lipid-related Effects of Resveratrol on Human Ectopic Endometrial Stromal Cells and a Rat Model of Endometriosis. Research Square. doi:10.21203/rs.3.rs$110546 / \mathrm{v} 1$

50. GuoL, TabrizchiR. Peroxisome proliferator-activated receptor gamma as adrug target in the pathogenesis of insulin resistance. Pharmacol Ther. 2006;111(1):145-173.

51. KennedyS, BergqvistA, ChapronC, etal. ESHRE guideline for the diagnosis and treatment of endometriosis. Hum Reprod. 2005;20 (10):2698-2704. doi:10.1093/humrep/dei135

52. BallardKD, SeamanHE, de VriesCS, etal. Can symptomatology help in the diagnosis of endometriosis? Findings from anational casecontrol study-part 1. Bjog. 2008;115(11):1382-1391. doi:10.11 11/j.1471-0528.2008.01878.x

53. WallerKG, LindsayP, CurtisP, etal. The prevalence of endometriosis in women with infertile partners. Eur JObstet Gynecol Reprod Biol. 1993;48(2):135-139. doi:10.1016/0028-2243(93)90254-A

54. SeamanHE, BallardKD, WrightJT, etal. Endometriosis and its coexistence with irritable bowel syndrome and pelvic inflammatory disease: findings from anational case-control study-part 2. Bjog. 2008;115(11):1392-1396. doi:10.1111/j.1471-0528.2008.01879.x

55. DullAM, MogaMA, DimienescuOG, etal. Therapeutic approaches of resveratrol on endometriosis via anti-inflammatory and anti-angiogenic pathways. Molecules. 2019;24(4):667. doi:10.3390/ molecules 24040667 
56. ChenFY, WangX, TangR-Y, etal. New therapeutic approaches for endometriosis besides hormonal therapy. Chin Med J(Engl). 2019;132(24):2984-2993. doi:10.1097/CM9.0000000000000569

57. KhazaeiMR, RashidiZ, ChobsazF, etal. Inhibitory effect of resveratrol on the growth and angiogenesis of human endometrial tissue in an invitro three-dimensional model of endometriosis. Reprod Biol. 2020;20(4):484-490. doi:10.1016/j.repbio.2020.07.012

58. BódisK, RodenM. Energy metabolism of white adipose tissue and insulin resistance in humans. Eur JClin Invest. 2018;48(11):e13017. doi:10.1111/eci.13017
59. WagnerN, WagnerKD. PPARs and angiogenesis-implications in pathology. IntJMol Sci. 2020;21(16):5723. doi:10.3390/ijms211 65723

60. YoshinoO, OsugaY, HirotaY, etal. Possible pathophysiological roles of mitogen-activated protein kinases (MAPKs) in endometriosis. Am JReprod Immunol. 2004;52(5):306-311. doi:10.1111/j.1600-0897.20 04.00231.x

\section{Publish your work in this journal}

Drug Design, Development and Therapy is an international, peerreviewed open-access journal that spans the spectrum of drug design and development through to clinical applications. Clinical outcomes, patient safety, and programs for the development and effective, safe, and sustained use of medicines are a feature of the journal, which has also been accepted for indexing on PubMed Central. The manuscript management system is completely online and includes a very quick and fair peer-review system, which is all easy to use. Visit http://www. dovepress.com/testimonials.php to read real quotes from published authors.

Submit your manuscript here: https://www.dovepress.com/drug-design-development-and-therapy-journal 